\title{
Endocarditis caused by rhodotorula successfully treated with 5-fluorocytosine
}

\author{
Yehezkel Naveh, Abraham Friedman, David Merzbach, and Nehama Hashman \\ From Departments of Paediatrics ' $B$ ' and Bacteriology, Rambam University Hospital, Aba Khoushy Medical \\ School, Haifa, Israel
}

A 7-year-old boy suffering from aortic regurgitation and mitral stenosis and regurgitation was admitted with endocarditis caused by Rhodotorula pilimanae and was treated successfully with orally administered antifungal agent, namely 5-fluorocytosine (5-FC). A dose of $100 \mathrm{mg}$ per $\mathrm{kg}$ body weight, divided into four equal parts, was prescribed. After a prolonged febrile period his temperature dropped to normal on the fourth day of 5-FC therapy. Review of the published reports disclosed few cases of endocarditis due to Rhodotorula spp. and this case seems to be the first treated with 5-FC. Follow-up in one year, after discharge from the hospital, revealed no evidence of relapse.

In recent years, the vast use of massive broad spectrum antibiotics, corticosteroids, cytotoxic drugs, narcotic drugs, and indwelling intravenous catheters in intravenous hyperalimentation, and the development of open-heart surgery resulted in the increase of fungal infections including fungal endocarditis. The majority of these infections are caused by candida species and Cryptococcus neoformans. 'Nonpathogenic' fungi play a smaller role but they have been found with increasing frequency. Review of the published material revealed few cases of rhodotorula endocarditis (Louria, Greenberg, and Molander, 1960; Shelburne and Carey, 1962; Leeber and Scher, 1969; Cherubin and Neu, 197I). We have recently treated such a case with 5fluorocytosine (5-FC), and because of the favourable outcome we felt that its presentation would be of value.

\section{Case report}

A 7-year-old boy was admitted to the paediatric department, with fever, headache, and abdominal pain of 6 days' duration.

Past history was uneventful except for recurrent tonsillitis and tonsillectomy two years before. No history of rheumatic fever or dental extractions could be elicited.

Physical examination revealed a pale well-nourished boy. His weight $22.5 \mathrm{~kg}$, his temperature $39.7^{\circ}$, his pulse $100 / \mathrm{min}$ regular and Corrigan type, and his blood pressure ro5/35 mmHg. The lungs were clear. The heart was mildly enlarged. No thrills were palpable.
Cardiac impulse was maximal at the apex, and visible outward pulsations at that area were noted.

At the apex, $M_{1}$ was diminished and a third sound was heard. A grade $3 / 6$ pansystolic murmur radiating to the left axilla and a grade $1 / 6$ short diastolic murmur were heard as well. At Erb's area, a grade 2/6 ejection type systolic murmur and a grade $\mathrm{I} / 6$ early diastolic murmur were heard. The liver was palpable $3 \mathrm{~cm}$ below the right costal margin but the spleen was not palpable. No joint involvement was observed on admission, but on the following day he complained of pain in the knees and ankles, and the left ankle was mildly swollen and hot. These complaints continued for three days. Funduscopic examination was normal.

Laboratory examination revealed a haemoglobin of $9 \mathrm{~g} / 100 \mathrm{ml}$, with slight hypochromia, and white blood cell count (WBC) of $19,400 / \mathrm{mm}^{3}$ of which 3 per cent were band forms and 78 per cent were segmented neutrophils. Urine analysis normal. Throat swab negative. Erythrocyte sedimentation rate (ESR) $125 \mathrm{~mm}$ in the first hour (Westergren). Blood urea nitrogen $23 \mathrm{mg} / \mathrm{roO}$ $\mathrm{ml}$. Total protein was $7 \cdot 1 \mathrm{~g} / \mathrm{1} 00 \mathrm{ml}$, of which albumin was $3.9 \mathrm{~g} / 100 \mathrm{ml}$ and globulin was $3.2 \mathrm{~g} / 100 \mathrm{ml}$. Blood serological tests were negative. C-reactive protein +++ . Antistreptolysin-O titre was 256 Todd units and 512 three days later. Multiple blood and urine cultures were negative.

An electrocardiogram showed normal sinus rhythm, normal $P R$ interval, $Q R S$ axis $+30^{\circ}$, and signs of hypertrophy of left atrium and ventricle. Chest $x$-ray disclosed an enlarged heart silhouette, with straightening of the left border, raised left main bronchus, and signs of venocapillary hypertension. The barium-filled oesophagus was indented by the left atrium and there was enlargement of the left ventricle. 


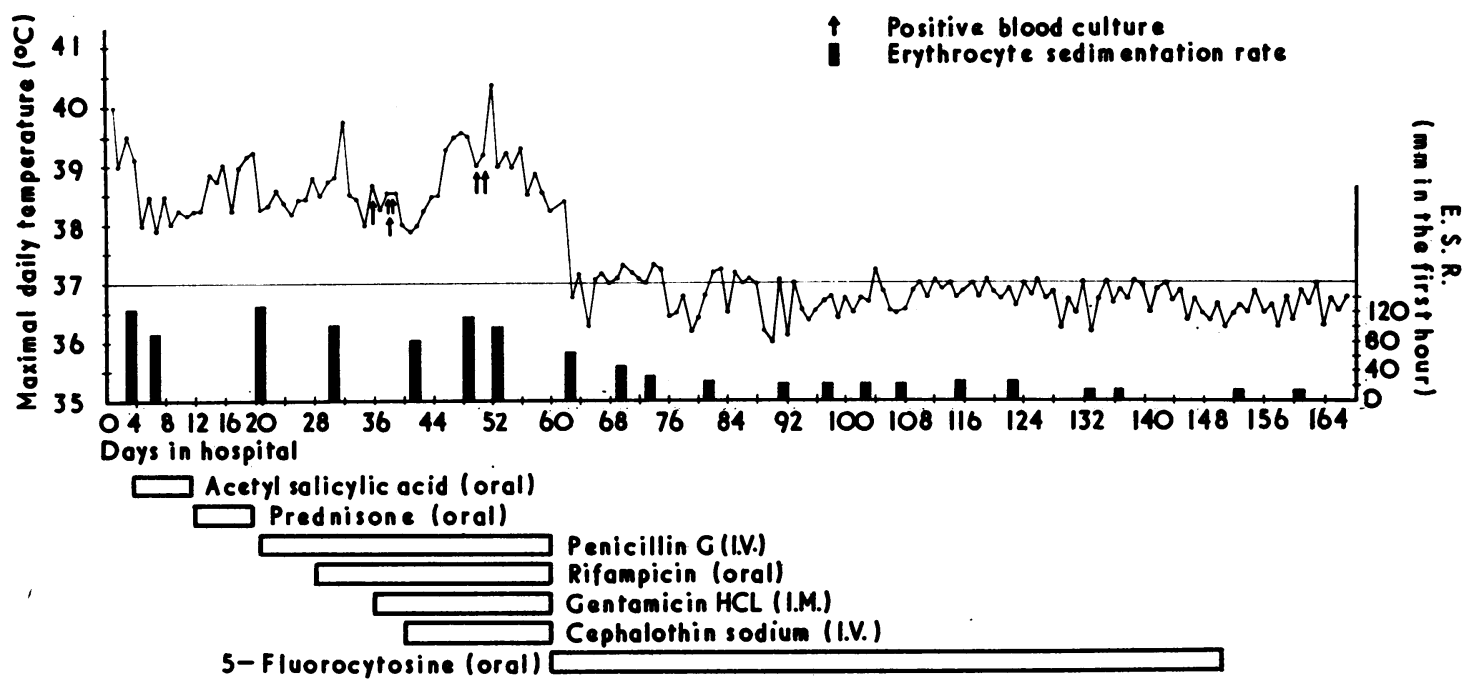

FIG. Patient's febrile course, erythrocyte sedimentation rate, and therapy.

A presumptive diagnosis of rheumatic fever with aortic regurgitation and mitral stenosis and regurgitation was made and he was put on acetylsalicylic acid $2 \mathrm{~g}$ daily (Fig.). Prednisone was initially avoided because of the presence of chicken-pox in the ward. No response to acetylsalicylic acid was obtained and it had to be replaced by prednisone $45 \mathrm{mg}$ daily, and the response to this was disappointing too. Consequently a suspicion of endocarditis was aroused and additional numerous blood cultures and bone-marrow culture were taken using different culture media. At the same time signs of incipient heart failure appeared and digoxin was given. The diagnosis of endocarditis was supported later by splenic enlargement, the appearance of a seagull murmur, and haematuria without granulated cylinders.

Therapy of penicillin G, 20 million units daily, was prescribed, followed by the graduated addition of rifampicin, $450 \mathrm{mg}$ daily, gentamacin hydrochloride, $60 \mathrm{mg}$ daily, and cephalothin sodium, $2 \mathbf{g}$ daily (Fig.). Indwelling venous catheters were not used.

Despite this intensive antibiotic treatment, his fever continued unremittingly, his clinical condition worsened, and his erythrocyte sedimentation remained maximal.

The diagnostic problem was resolved when a fungus, initially disregarded as technical contamination and identified later as Rhodotorula pilimanae, was isolated from six specimens of blood taken at different occasions over a period of 16 days (Fig.).

From that day, all antibiotics were stopped and 5fluorocytosine ( $5-\mathrm{FC}) 2 \mathrm{~g}$ (100 $\mathrm{mg} / \mathrm{kg}$ body weight) daily divided into 4 equal parts was prescribed. Three days later, his temperature dropped to normal for the first time since admission. His erythrocyte sedimentation rate returned to normal two months later.

5-FC was continued for three months without evidence of toxic effects in repeated blood counts and results of liver and kidney function tests. Attempts to deter- mine the blood level of 5-FC failed because of technical difficulties. Blood cultures during and after 5-FC therapy were negative.

The patient was discharged clinically well with maintenance of digoxin and preventive benzathine penicillin.

Periodic clinical evaluations in the follow-up of one year have revealed no evidence of recurrent fungal infection.

\section{Mycology}

Preliminary isolation Blood cultures were observed daily according to the laboratory routine on brain heart infusion (Difco). On various occasions other media were also used: thioglycollate broth, cooked meat broth (Robertson), and mycoplasma broth medium (Hayflick). No bacteria were isolated in these examinations.

In 6 blood specimens macroscopical appearance of growth was observed at the bottom of the media after 3 to 5 days. Microscopical examination revealed budding yeast cells of spherical or ovoidal shape. No zone of a capsule was detected in India ink preparations. Subcultures on plates of blood agar and Sabouraud dextrose agar were incubated at $37^{\circ} \mathrm{C}$ and at room temperature. Slimy colonies with pink-orange pigment developed within 3 days at both temperatures; the size of the colonies increased and the pigment intensified on further incubation. No hyphae were seen. Urease was produced on Cristensen's agar. These results indicated the isolation of yeast cells, probably belonging to the genus cryptococcus or rhodotorula.

Biochemical identification Dextrose, sucrose, maltose, and lactose were not fermented after 4 weeks. Assimilation of carbohydrates was examined on bacto yeast nitrogen base (Difco) and read after 7 and 24 days: dextrose, sucrose, and raffinose were assimilated; 
lactose, maltose, inositol, melibiose, and melezitose were not assimilated. Nitrate nitrogen, tested on bacto yeast carbon base (Difco), was not utilized.

Animal test $0.5 \mathrm{ml}$ of a 5-day culture broth was injected intraperitoneally into 5 mice. No response was observed within 4 weeks.

According to the above characteristics and taxonomic criteria (van der Walt, 1970; Phaff and Fell, 1970; Phaff and Ahearn, 1970), all 6 cultures were identified as Rhodotorula pilimanae.

\section{Discussion}

The absence of history of rheumatic heart disease and the fact that his present illness does not fulfil the Jones' criteria for diagnosing rheumatic fever do not enable us to determine positively a rheumatic background of his illness. In any event, there is no doubt that the child had on admission physical findings of aortic regurgitation and mitral stenosis and regurgitation and $x$-ray and electrocardiographic findings supporting left atrial and ventricular hypertrophy. The $x$-ray and electrocardiographic findings prove that the child had had valvular involvement for a prolonged period, and that Rhodotorula spp. was therefore superimposed upon previously damaged aortic and mitral valves.

The continuity of high fever (Fig.), despite acetylsalicyclic acid and prednisone administration, supports the diagnosis of endocarditis from the beginning of admission. It is controversial whether the endocarditis was primarily fungal or began as a bacterial endocarditis and by the influence of corticosteroid and/or antibiotic administration, fungal endocarditis developed. The combination of a clinical picture of subacute bacterial endocarditis with the absence of bacterial growth in blood cultures, the fact that Rhodotorula pilimanae was the single organism isolated repeatedly from blood cultures over a period of 16 days, and the continuity of fever despite administration of massive broad spectrum antibiotics gives weight to the diagnosis of primary fungal endocarditis. The fact that the fungus was not grown on the first month of hospitalization could be attributed to low grade fungaemia which increased after antibiotic therapy. Nevertheless, secondary fungaemia could not be excluded.

Review of 656 cases of infective endocarditis (Cherubin and Neu, I97I) revealed only one case of endocarditis caused by Rhodotorula spp. Recently (Jennings and Bennet, 1972) Rhodotorula $s p p$. isolates were found in 57 out of 8062 specimens, comprising $0.7 \mathrm{I}$ per cent. The specimens were obtained from clinical routine sources, mainly urine, stool, blood, bone marrow, respiratory tract, skin, throat, and wound examinations. None of these isolates appeared to be the cause of any infection. Stool was the commonest single source which showed the highest incidence of Rhodotorula spp. $(2 \cdot 6 \%)$. Rhodotorula pilimanae was isolated only on one occasion.

It is probable that in our case the source of the strain causing endocarditis was endogenous.

The clinical picture of infections caused by opportunistic fungi not ordinarily pathogenic such as rhodotorula, species of torulopsis, species of candida, and aspergillus may vary from mild to severe and in such case may simulate Gram-negative septicaemia (Louria et al., 1960).

We do not think that there is any causal relation between stopping of intravenous fluids and antibiotic treatment and dropping of fever and erythrocyte sedimentation rate to normal since - a) the boy had had high fever and high erythrocyte sedimentation rate before administration of intravenous fluids and antibiotic therapy, and $b$ ) the fungus was isolated from blood cultures taken during 16 days and in this period drug vials were changed frequently and infusion sets were changed every two days or less. Therefore, reduction of fever and erythrocyte sedimentation rate could be the result of efficient treatment only.

$5-\mathrm{FC}$ is a relatively new fluoro-pyrimidine antimycotic agent which has the advantage of oral administration given in a dose of 50 to $150 \mathrm{mg}$ per kg body weight. 5-FC has antimycotic activity against most strains of Cryptococcus neoformans and Candida species (Fass and Perkins, 1971 ; Utz, 1972) but has not been tried yet in rhodotorula infection Experience was especially acquired in candidiasis and cryptococcal meningitis (Tassel and Madoff, 1968; Fass and Perkins, 1971; Steer et al., 1972; Isacson et al., 1972). Published treatment of fungal endocarditis with 5-FC is limited to about 5 cases (Record et al., 197I ; Fass and Perkins, 197I; Steer et al., 1972). There are no clear guidelines yet as to the duration of therapy for treating fungal endocarditis with 5-FC. We preferred a course of three months in order to prevent a possible relapse. Yet, one must bear in mind that prolonged treatment with high doses of 5-FC may be dangerous (Record et al., 1971).

Lastly, it should be emphasized that early recognition of the possibility of fungal infection in severely ill patients with unexplained fever, especially those getting intravenous fluids with or without antibiotics, corticosteroids, or cytotoxic drugs, may be life saving.

We wish to thank Dr. Norberto Roguin, and Dr. Lionel Davidson, who assisted in the preparation of this paper. 


\section{References}

Cherubin, C. E., and Neu, H. C. (197I). Infective endocarditis at the Presbyterian hospital in New York City from 19381967. American fournal of Medicine, 51, 83.

Fass, R. J., and Perkins, R. L. (1971). 5-Fluorocytosine in the treatment of cryptococcal and candida mycoses. Annals of Internal Medicine, 74, 535.

Isacson, M., Noah, Z., Faber, J., Herishano, Y., and Gottfried, L. (1972). Use of 5-fluorocytosine in systemic candidiasis in infancy. Archives of Disease in Childhood, 47, 954.

Jennings, A. E., and Bennet, J. E. (1972). The isolation of red yeast-like fungi in a diagnostic laboratory. fournal of Medical Microbiology, 5, 39r.

Leeber, D. A., and Scher, I. (1969). Rhodotorula fungemia presenting as 'endotoxic' shock. Archives of Internal Medicine, $123,78$.

Louria, D. B., Greenberg, S. M., and Molander, D. W. (1960). Fungemia caused by certain nonpathogenic strains of the family cryptococcaceae. Nerw England fournal of Medicine, 263, I28I.

Phaff, H. J., and Ahearn, D. G. (1970). Rhodotorula Harrison. In The Yeasts: $A$ Taxonomic Study, p. 1187. Ed. by J. Lodder. North Holland Publishing Company, Amsterdam and London.

Phaff, H. J., and Fell, J. W. (1970). Cryptococcus Kützing emend. Phaff et Spencer. In The Yeasts: A Taxonomic
Study, p. 1088. Ed. by J. Lodder. North Holland Publishing Company, Amsterdam and London.

Record, C. O., Skinner, J. M., Sleight, P., and Speller, D. C. E. (197I). Candida endocarditis treated with 5fluorocytosine. British Medical fournal, r, 262.

Shelburne, P. F., and Carey, R. J. (1962). Rhodotorula fungemia complicating staphylococcal endocarditis. Fournal of the American Medical Association, 180, 38.

Steer, P. L., Marks, M. I., Klite, P. D., and Eickhoff, T. C. (1972). 5-Fluorocytosine: an oral antifungal compound. A report on clinical and laboratory experience. Annals of Internal Medicine, 76, I5.

Tassel, D., and Madoff, M. A. (1968). Treatment of Candida sepsis and Cryptococcus meningitis with 5 -fluorocytosine. fournal of the American Medical Association, 206, 830.

Utz, J. P. (1972). Flucytosine. New England fournal of Medicine, 286, 777.

van der Walt, J. P. (1970). Criteria and methods used in classification. In The Yeasts: A Taxonomic Study, p. 34. Ed. by J. Lodder. North Holland Publishing Company, Amsterdam and London.

Requests for reprints to Dr. Y. Naveh, Department of Paediatrics ' $B$ ', Rambam University Hospital, Haifa, Israel. 